\title{
A new approach to the reduction of "Carte du Ciel" plates
}

\author{
A. Ortiz-Gil ${ }^{1, \star}$, M. Hiesgen ${ }^{2}$, and P. Brosche ${ }^{1}$ \\ 1 Sternwarte der Universität Bonn, Auf dem Hügel 71, D-53121 Bonn, Germany \\ 2 Observatoire de Strasbourg, 11 rue de l'Université, 67000 Strasbourg, France
}

Received May 20; accepted August 4, 1997

\begin{abstract}
A new procedure for the reduction of "Carte du Ciel" plates is presented. A typical "Carte du Ciel" plate corresponding to the Bordeaux zone has been taken as an example. It shows triple exposures for each object and the modeling of the data has been performed by means of a non-linear least squares fitting of the sum of three bivariate Gaussian distributions. A number of solutions for the problems present in this kind of plates (optical aberrations, adjacency photographic effects, presence of grid lines, emulsion saturation) have been investigated. An internal accuracy of 0.1 in $x$ and $y$ was obtained for the position of each of the individual exposures. The external reduction to a catalogue led to results with an accuracy of $0^{\prime \prime} 16$ in $x$ and $0^{\prime \prime} 13$ in $y$ for the mean position of the three exposures. A photometric calibration has also been performed and magnitudes were determined with an accuracy of 0 m.09.
\end{abstract}

Key words: astrometry - methods: data analysis open cluster: M 67

\section{Introduction}

The "Carte du Ciel" (CdC in what follows) was one of the first large international joint astronomical projects of the history. It was officially initiated in 1887 with a double target: the construction of a sky catalogue complete down to the $11^{\text {th }}$ magnitude (which eventually became the Astrographic Catalogue) and the construction of a sky chart down to the $14^{\text {th }}$ magnitude. These projects represent the very first photographic registers of the whole sky. In consequence, they constitute a very important source of data for proper motion studies (see, for example, Geffert et al. 1996) because of the large time interval when comparing with today observations (about one hundred years

Send offprint requests to: A. Ortiz-Gil

* Present address: Dept. of Astrophysics \& Optics, School of Physics, The University of New South Wales, Sydney 2052, NSW, Australia. in most of the cases) as well as for studies on galactic structure and kinematics.

Part of the data was completely measured and the results were published as "Astrographic Catalogue". A re-reduction with these published data has been recently performed by Urban \& Corbin (1996). Other groups are taking a complete $\mathrm{CdC}$ second epoch plates (Potter et al. 1996). On the other hand, Carte plates have never been systematically measured because they were not intended to give positions but only to be a sky chart. But due to the deeper images taken (we have identified stars fainter than $B=15$ (Hiesgen et al. in preparation) these plates are of great importance because of the large amount of data they contain and the time elapsed since they were produced.

The plates were exposed three times, with the exposures being placed at the vertices of an approximately equilateral triangle (see Fig. 1). They were also provided with a grid of perpendicular lines in order to ease the star position measurements. These two characteristics make the accurate measurement of positions very difficult in some specific cases because of: 1) merging of the three images when the star is brighter than $B \approx 12$ and then the presence of an adjacency photographic effect called "Kostinsky effect" (Kostinsky 1907; Ross 1921) which increases the measured distance between the exposures, and 2) stars lying on a grid line or close enough to let the Kostinsky effect show up. Optical aberrations (mainly spherical aberration and field curvature) are also present.

These problems have prevented astrometrists till now from using these triple image plates as a valuable source of data, although there are some punctual exceptions (see Geffert et al. 1996, for a recent example). In this paper our aim is to show that good astrometric and photometric results are achievable, following the general procedure outlined in this work. A different procedure was proposed by Bonnefond (1991) and used by Geffert et al. (1996). Our method is able to deal with stars brighter than the upper limit of $V=9.5$ in Geffert et al. (1996) because it can work with blended images. 
A European Community Human and Capital Mobility Network was initiated in 1994 under the name of "Salvaging an Astrometric Treasure" (Ortiz-Gil et al. 1995; Hiesgen et al. 1996) with the scope of measuring these plates and using the data for a variety of applications, such as extension of the Hipparcos reference system to fainter magnitudes, determination of proper motions with an accuracy of $210^{-3} \operatorname{arcsec} / y r$, proper motions of globular and open clusters, photographic magnitudes with an accuracy of about 0.1 , or studies related to galactic structure and kinematics.

Different typical CdC plates have been analysed in order to study how to deal with the specific problems which arise and may vary much from plate to plate. Therefore, a complete study of the characteristics of each individual plate is necessary. Here we present the complete analysis performed with a plate which was gently provided by Bordeaux Observatory (France). It was taken in February 1922 and covers a $2^{\circ} \times 2^{\circ}$ field with centre at $\alpha=8^{\mathrm{h}} 50^{\mathrm{m}}$ and $\delta=11^{\circ} 48^{\prime}$ (1900 equinox). This field is interesting because it includes the open cluster M 67 . It shows three exposures (Fig. 1), placed at the vertices of a triangle.

The plate was scanned with the PDS $2020 \mathrm{GM}^{\text {plus }}$ microdensitometer of the Astronomical Institute Münster (Germany). In the process of scanning the plate on-line search and segmentation techniques were employed, which produced an output file containing detected objects in the form of "picture frames" of individual images (Horstmann $1988)$. These frames have a size of $61 \times 61$ pixels $(0.61 \times$ $0.61 \mathrm{~mm})$, or $81 \times 81$ pixels $(0.81 \times 0.81 \mathrm{~mm})$ in the case of bright stars, to assure that all three exposures belonging to the same triangle are included in the frame.

Visual inspection of these frames allows us to remove from the set those frames in which any of the three exposures is missing, there is confusion between exposures due to the presence of double stars or the system has identified a spurious image. This implies that we can end up with at most three different frames corresponding to the same triplet. They are identified by their equal positions on the plate and the one which will be finally used in the study of the plate characteristics and reduction will be the one giving a better fit to the triple Gaussian model described later.

In the rest of this work we have assigned the following numbers to the three exposures: exposure 1 (top left exposure in Fig. 1), exposure 2 (top right exposure in Fig. 1) and exposure 3 (bottom exposure in Fig. 1).

\section{Modeling the data}

To calculate the positions of each of the components of these triple images the fitting with a model consisting on the sum of three bivariate Gaussian distributions was applied (Dick et al. 1993). The density $D_{i, j}$, measured in

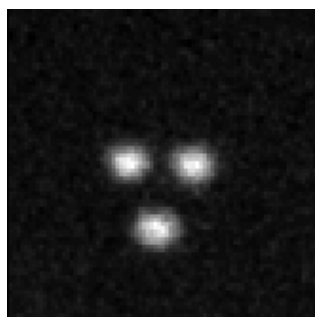

Fig. 1. One star as example for the triple exposures on the $\mathrm{CdC}$ plates

pixel $i, j$, is then:

$$
\begin{aligned}
D_{i j}= & B+\sum_{k=1}^{3} A_{k} \exp \left\{-\frac{1}{2}\left[\frac { 1 } { 1 - t _ { k } ^ { 2 } } \left(\left(\frac{x_{i j}-x_{c k}}{\sigma_{x k}}\right)^{2}\right.\right.\right. \\
& \left.\left.\left.+\left(\frac{y_{i j}-y_{c k}}{\sigma_{y k}}\right)^{2}-2 t_{k}\left(\frac{x_{i j}-x_{c k}}{\sigma_{x k}}\right)\left(\frac{y_{i j}-y_{c k}}{\sigma_{y k}}\right)\right)\right]^{s_{k}}\right\} .
\end{aligned}
$$

In this expression $B$ is the background of the frame. As the triple images are confined to areas of $0.81 \times 0.81 \mathrm{~mm}$ at most, $B$ can be considered constant in each of these frames (see Sect. 6). $A_{k}$ is the peak density of the $k$-th image, while $x_{i j}, y_{i j}$ are the pixel coordinates and $x_{c k}, y_{c k}$ are the centre coordinates of the $k$-th image. $\sigma_{x k}, \sigma_{y k}$ and $t_{k}$ are the parameters of an ellipse with arbitrary orientation and axis size. These three parameters are related to the ellipse semimajor and semiminor axis, $a_{k}$ and $b_{k}$, by the expressions:

$a_{k}=\left\{\frac{1}{2}\left[\left(\sigma_{x k}^{2}+\sigma_{y k}^{2}\right)+\sqrt{\left(\sigma_{x k}^{2}-\sigma_{y k}^{2}\right)^{2}+4 t_{k}^{2} \sigma_{x k}^{2} \sigma_{y k}^{2}}\right]\right\}^{\frac{1}{2}}$
$b_{k}=\left\{\frac{1}{2}\left[\left(\sigma_{x k}^{2}+\sigma_{y k}^{2}\right)-\sqrt{\left(\sigma_{x k}^{2}-\sigma_{y k}^{2}\right)^{2}+4 t_{k}^{2} \sigma_{x k}^{2} \sigma_{y k}^{2}}\right]\right\}^{\frac{1}{2}}$

and the angle between $X$ and $a_{k}$ axes is

$\theta_{k}=\operatorname{arcos} \frac{1}{\sqrt{1+\left(\frac{\lambda_{k}-\sigma_{x k}^{2}}{t_{k} \sigma_{x k} \sigma_{y k}}\right)^{2}}}$

where

$\lambda_{k}=\frac{1}{2}\left[\left(\sigma_{x k}^{2}+\sigma_{y k}^{2}\right)+\sqrt{\left(\sigma_{x k}^{2}-\sigma_{y k}^{2}\right)^{2}+4 t_{k}^{2} \sigma_{x k}^{2} \sigma_{y k}^{2}}\right]$.

The parameter $s_{k}$ is a flattening parameter which takes into account the saturation of the photographic emulsion.

The fitting process was performed by means of the Levenberg-Marquardt method (Marquardt 1963) for nonlinear least squares fitting to work out the values of the 22 free parameters in the model. The fitting was applied to small frames of $0.61 \times 0.61 \mathrm{~mm}(61 \times 61$ pixels $)$, or $0.81 \times 0.81 \mathrm{~mm}(81 \times 81$ pixels $)$ for bright stars, around each set of triple images. One of our main objectives is to reduce the number of these free parameters in order to achieve better results (see Sect. 6). At the moment this software has not been fully prepared for its use by other groups, but we intend to make it available to the community as soon as possible. 


\section{Optical aberrations}

Four optical aberrations would mainly be expected to show up in an optical system of the kind used in the $\mathrm{CdC}$ astrographs. These are coma, spherical aberration, field curvature and chromatic aberration. In particular, coma makes the images to become asymmetric as it gives them a comet-like appearance. Spherical aberration and field curvature generate extended and elongated images, respectively, from pointlike ones. How much elongated and asymmetric the images are is of importance in order to see how the optical aberrations are influencing the final results in the plate reduction.

\subsection{Ellipticity}

Images at a radial distance from the plate centre greater than $\approx 5 \mathrm{~cm}$ show a significant ellipticity, which is defined as:

$e_{k}=1-\frac{b_{k}}{a_{k}}$

where $a_{k}, b_{k}$ are the image semimajor and semiminor axes of exposure $k$, respectively, given by Eqs. (2) and (3).

In Fig. 2 the ellipticity as a function of the plate position is shown. Segment lengths are proportional to the mean ellipticity of the three exposures, while their orientation with respect to the $X$ axis is the mean of the three angles $\theta_{k}$. We expect to encounter this effect in all $\mathrm{CdC}$ plates because they all were taken with a similar kind of optics. Two examples of these images are shown in Fig. 3.

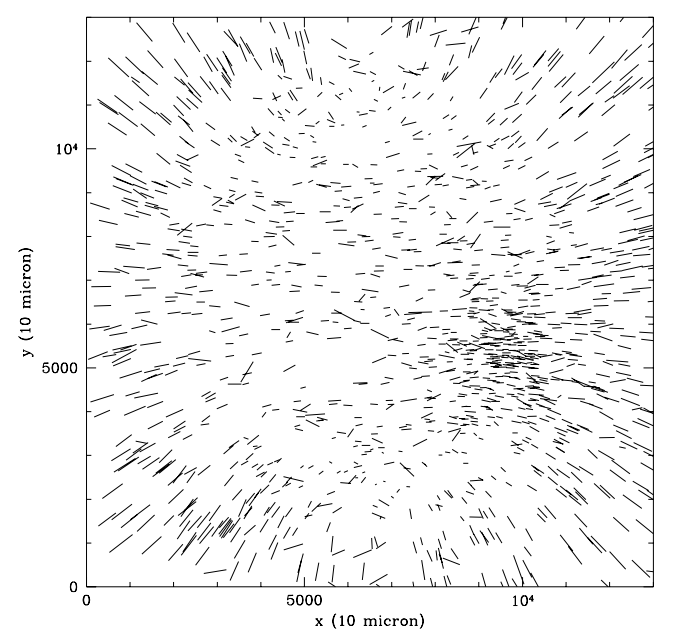

Fig. 2. Field curvature in the plate. The length of the lines is proportional to the mean ellipticity of the three exposures of each stellar image. The orientation with respect to the $x$-axis is proportional to the mean of the three angles $\theta_{k}$
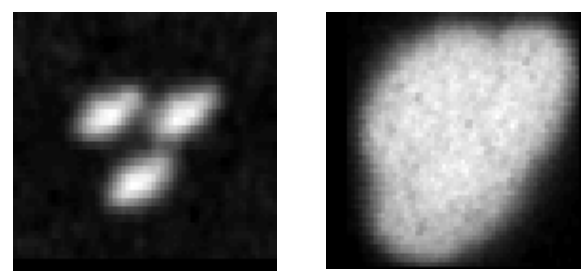

Fig. 3. Effects of optical aberrations are shown for two stars of different magnitudes

No significant dependence of the position error on the ellipticity can be found after performing the plate reduction with the external catalogue (Fig. 4). This suggests that the model is therefore able to deal correctly with elongated images.

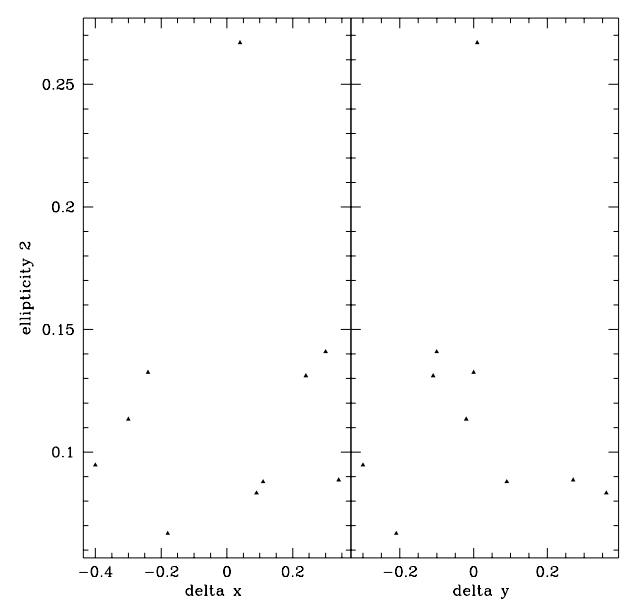

Fig. 4. Image ellipticities as a function of the residuals in $x$ and $y$ for the three exposures after performing the plate reduction with an external catalogue. No dependence on the ellipticity value is evident here. Ellipticity values of exposure 2 have been used as an example. Analog diagrams are obtained with the ellipticity values of exposures 1 and 3

\subsection{Image asymmetry}

A study on the asymmetry of the images in the plate has been carried out by means of analysing the skewness of the marginal distribution along the "possible" asymmetry axis:

skew $=\frac{\sum_{i} f\left(x_{i}\right)\left(x_{i}-\bar{x}\right)^{3} / \sum_{i} f\left(x_{i}\right)}{\sigma^{3}}$

where $f\left(x_{i}\right)$ is the marginal distribution along the image semimajor axis and $\sigma$ stands for the marginal distribution standard deviation.

Most of the skewness values $(88.7 \%)$ lie on the interval $[-0.2,0.2]$ (Fig. 5), which seems to indicate that the images can be considered symmetric and, in consequence, 


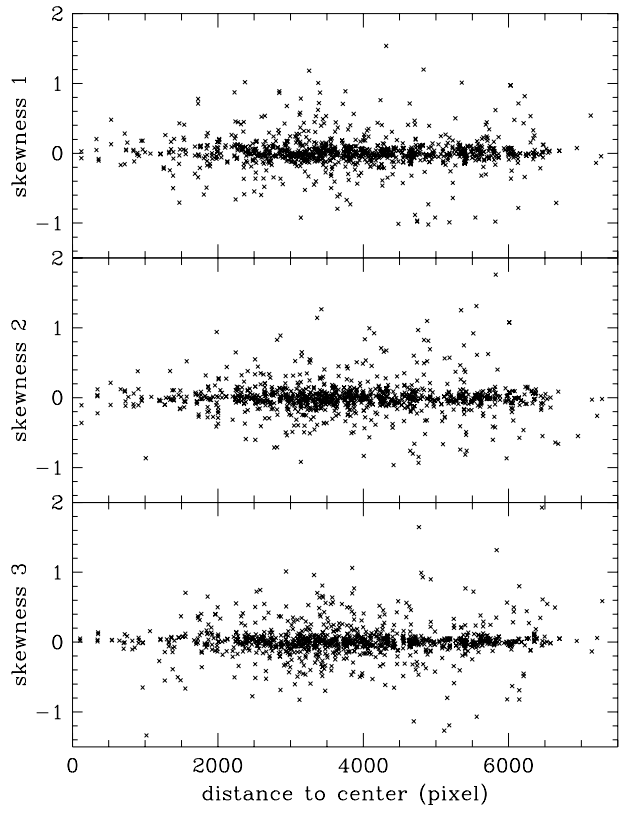

Fig. 5. Skewness values as a function of the distance to the plate centre. $88.7 \%$ of the values lie within $[-0.2,0.2]$

that coma is not the dominant cause for the distortion but rather the field curvature and spherical aberration. The last one makes the pointlike images to appear as disks while the first one is responsible for the elongation of these disks.

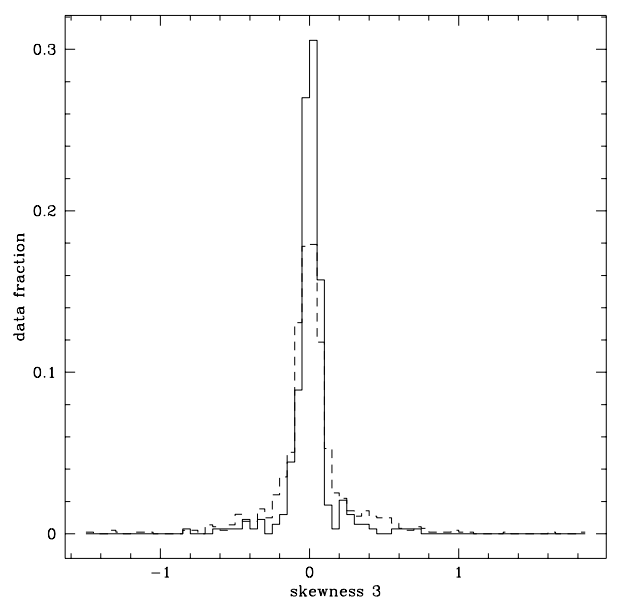

Fig. 6. Histograms of skewness values for stars fainter (dashed line) and brighter (solid line) than $B \approx 13.5$. The histogram for the fainter stars is broader than the one for brighter stars

There are two other facts which reinforce the conclusion that asymmetry has no influence on the data:

1) The largest skewness values correspond to the fainter images, which are much sensitive to plate defects and noise which might alter their density profile. Figure 6 shows the histograms for the skewnesses of stars with mag- nitude $B \gtrsim 13.5$ and $B \lesssim 13.5$. The standard deviation of both histograms has been calculated and the results show that the distribution for the brightest stars around 0 is narrower than the corresponding one for the faintest ones, as one can see from Table 1. At the bright end the non-linear response of the plate also contributes to this effect as the stars are increasingly broader thus masking features visible in fainter star images.

Table 1. Distribution of skewness values around zero

\begin{tabular}{lllll}
\hline$B$ magnitude $^{a}$ & data $^{b}$ & $\sigma_{1}{ }^{c}$ & $\sigma_{2}{ }^{d}$ & $\sigma_{3}{ }^{e}$ \\
\hline$\lesssim 13.5$ & 337 & 0.16 & 0.16 & 0.15 \\
$\gtrsim 13.5$ & 910 & 0.25 & 0.30 & 0.27 \\
\hline
\end{tabular}

${ }^{a}$ magnitude interval considered

${ }^{b}$ number of stars used in each case

$c, d, e$ standard deviations of the distributions of skewness values computed for exposures 1, 2 and 3, respectively.

This suggests that the images are symmetric and asymmetries only arise because of noise in faint stars. This is a normal effect in photographic plates and it is not a specific problem of the $\mathrm{CdC}$ plates.

2) The residuals for positions on the plate after the plate reduction with the catalogue (PPM) are not a function of the skewness values (Fig. 7).

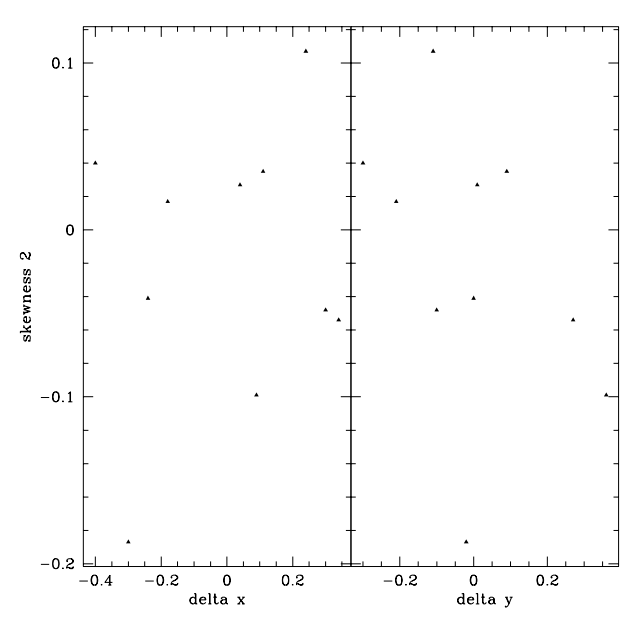

Fig. 7. Residuals for star positions after the plate reduction with an external catalogue vs. skewness values. Residuals are independent of the skewness. As an example we show here the skewness computed for exposure 2. Analog diagrams are obtained for skewness values of exposures 1 and 3

As a conclusion one can say that at least the asymmetry in the images is not important enough to have a detectable influence on the data. Anyway, it has been found that optical aberrations in $\mathrm{CdC}$ plates can be very 
complicated when the centres of the telescope lenses were misaligned, being dependent on magnitude and colour (Zacharias, private communication). And, as usual when working with photographic plates, plate borders are of lower quality than the plate central region as they introduce some trends in the final position residuals when performing the plate reduction.

\section{Astrometric reduction}

Further plate characteristics influencing the final astrometric accuracy have been found. The presence of a grid in the plates prevented us from considering stars lying on or very close to the lines (Fig. 8). Because of this we loose approximately $15 \%$ of the total number of stars in the plate. These lines introduce changes in the density of the images and in the positions of the nearby ones due to the Kostinsky effect (Kostinsky 1907; Ross 1921). This effect shows up also when images of stars lie very close to each other. The developer is exhausted in the zones where two images are in contact or very close and the amount of the products from the development process is larger there than in other zones. These products inhibit the chemical reaction which takes place during the development and the final result is an apparent repulsion of the images. Due to the small separation between the three exposures $(\approx 170 \mu \mathrm{m})$, they begin to merge in the case of stars of magnitude $B \lesssim 12$, and consequently, the Kostinsky effect introduces changes in the "true" positions of the images. These can be quite large: changes of $\approx 40 \mu \mathrm{m}$ have been detected (see Fig. 9). This effect is very difficult to model: it changes from plate to plate as it depends on the development process of each plate individually. The determination of its variation as a function of the stellar magnitude is one of the most important targets in order to be able to correct for it.

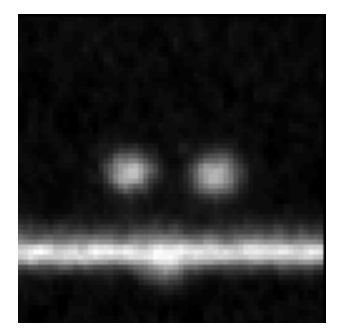

Fig. 8. Example of a triple star image of which one is on a grid line

\subsection{Plate-to-plate transformation}

To obtain the internal accuracy of the plate, we have transformed the positions of two of the three exposures to the reference system of the third one by means of a linear

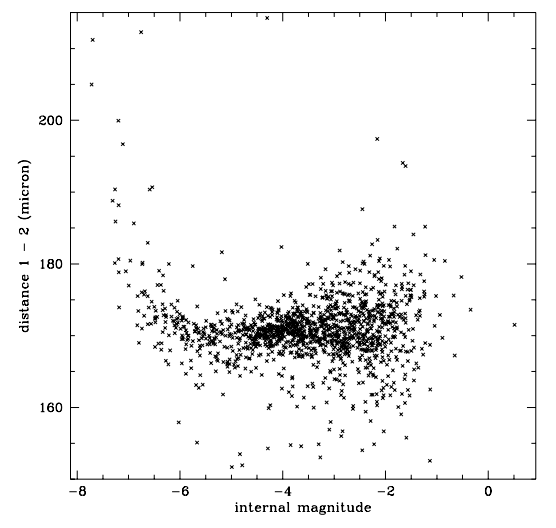

Fig. 9. Distance between exposures 1 and 2 of each star as a function of a measure of the star brightness. The apparent repulsion between exposures 1 and 2 due to the Kostinsky effect is more evident for the brightest stars

transformation, and the deviations from the new computed positions were obtained. It is equivalent to consider three different single exposure plates. The linear transformation was computed by least squares fitting of the positions of 552 stars in the field whose magnitudes ranged from $B=12$ to $B=14.5$ to avoid large errors introduced by very bright or very faint stars. Assuming that all exposures equally contribute to the deviations, the rms of these deviations can be calculated for each individual exposure using 1108 stars with $B \leq 15.5$ (thereby excluding the ten ones with larger errors). Since these plates were taken originally to be complete up to the $14^{\text {th }}$ magnitude, images fainter than $B \approx 15.5$ have poor quality. Almost all the stars with larger errors were fainter than $B=13$. The results are presented in the first row in Table 2.

Table 2. rms of the position resulting from the internal plate comparison

\begin{tabular}{lllll}
\hline field $^{a}$ & model $^{b}$ & $\sigma(\Delta x)^{c}$ & $\sigma(\Delta y)^{d}$ & no. of stars \\
\hline whole plate & 22 & $\pm 0^{\prime \prime} 16$ & $\pm 0^{\prime \prime} 16$ & 1108 \\
$1^{\circ} \times 1^{\circ}$ & 22 & $\pm 0^{\prime \prime} 12$ & $\pm 0^{\prime \prime} 08$ & 394 \\
plate centre & 22 & $\pm 0^{\prime \prime} 09$ & $\pm 0^{\prime \prime} 09$ & 648 \\
whole plate & 12 & $\pm 0^{\prime \prime} 15$ & $\pm 0^{\prime \prime} 12$ & 1108 \\
plate centre & 12 & $\pm 0^{\prime \prime} 10$ & $\pm 0^{\prime \prime} 10$ & 648 \\
\hline
\end{tabular}

${ }^{a}$ zone of the plate used for analysis

${ }^{b}$ number of free parameters in the model to determine the positions

${ }^{c, d} \mathrm{rms}$ of the position in $x, y$, respectively

${ }^{e}$ number of stars used.

This analysis was also performed over the smaller region of $1^{\circ} \times 1^{\circ}$ in the plate covering the zone in which the cluster M67 is located, since a good external catalogue 
is available for this area (Girard et al. 1989). The linear model was in this case computed using a total of 215 stars with magnitudes ranging from $B=12$ to $B=14.5$. The final $\mathrm{rms}$ were obtained from a set of 394 stars brighter than 15.5 (second row in Table 2). One can see that $\sigma(\Delta x)$ are larger than those for $\sigma(\Delta y)$. This may indicate that there are some kinds of local distortions which show up when studying only a small plate region, while they are absorbed by the model when considering the whole plate. They can be due to slight emulsion displacements during the plate drying process or because storage of the plate in vertical position for almost one hundred years, to small errors in the telescope tracking, or to optical distortions as the small field considered around M 67 is at the plate edge, where these distortions are expected to be stronger. Also the background, and consequently the noise, was found to be larger at the plate borders (see Fig. 12).

To determine if these "plate border effects" could account for the fact that $\sigma(\Delta x)>\sigma(\Delta y)$ an internal reduction using stars closer than $5 \mathrm{~cm}$ to the plate centre was carried out. Accuracies of $\sigma(\Delta x)=\sigma(\Delta y)=0$ ". 09 were found. This result clearly reflects the poorer quality of plate borders. Therefore, it seems that the triple Gaussian model can deal with this trend of $\sigma(\Delta x)>\sigma(\Delta y)$ when considering the whole plate, although the final accuracies are poorer than when only considering the plate central region.

\subsection{Plate-to-catalogue reduction}

For the $1^{\mathrm{o}} \times 1^{\mathrm{o}}$ region mentioned before containing the cluster a separate reduction was performed. The external comparison was carried out with an astrometric catalogue specifically built for this field (Girard et al. 1989). It has an external error of 0 "' 16 in the star positions for 1950.8, the catalogue weighted mean epoch. The cluster is located $10^{\prime}$ north of the field centre. The plate model used consisted of polynomials in $x$ and $y$ up to the second order and the reduction was performed using the mean position over the three positions corresponding to the three exposures we have per star. In this way it is possible to avoid to some degree the consequences of the Kostinsky effect over the brightest stars. The rms of the deviations are shown in the second row in Table 3 . In these calculations three stars with exceptionally large deviations were not taken into account and the star showing the largest deviation after the reduction was also rejected.

The reduction of the full plate was performed using the PPM as an external catalogue. Only 10 stars (with magnitudes $B=8.24 \leq B \leq 11.13$ ) could be used in the reduction as in the PPM stars are normally brighter than $V=11$. The triple images of these stars are quite blended in our plates and the errors for the parameters obtained in their Gaussian fitting are a bit worse than the ones corresponding to fainter stars. The plate model used consisted of polynomials in $x$ and $y$ up to the second order
Table 3. rms of the triplet mean position resulting from the plate reduction with an external catalog

\begin{tabular}{lllll}
\hline catalog $^{a}$ & model $^{b}$ & $\sigma(\Delta x)_{m}{ }^{c}$ & $\sigma(\Delta y)_{m}{ }^{d}$ & no. of stars \\
\hline PPM & 22 & $0^{\prime \prime} 23$ & $0^{\prime \prime} 19$ & 10 \\
Girard & 22 & $0^{\prime \prime} 20$ & $0^{\prime \prime} 20$ & 196 \\
PPM & 12 & $0^{\prime \prime} 16$ & $0^{\prime \prime} 13$ & 10 \\
\hline
\end{tabular}

${ }^{a}$ catalog employed

${ }^{b}$ number of free parameters in the model used to determine the star positions

${ }^{c, d} \mathrm{rms}$ of each triplet mean $x, y$ coordinates

${ }^{e}$ number of stars used.

and the rms of the deviations are shown in the first row in Table 3. The larger values for $\sigma(\Delta x)$ than for $\sigma(\Delta y)$ can be due to the fact that the reference stars happen to be in regions of the plate where the optical distortions are stronger along $x$-axis than $y$-axis and the fitting algorithm is slightly poorer in the case of the brightest stars because when the exposures merge the determination of the exposure centres is less accurate. Errors during the telescope tracking can also be an explanation.

In Fig. 10 the residuals in the mean position are plotted as a function of the plate position. They are uncorrelated so it seems that there are no global systematic errors present in the plate although ten reference stars are clearly too few to categorically affirm that there are no systematic errors left.

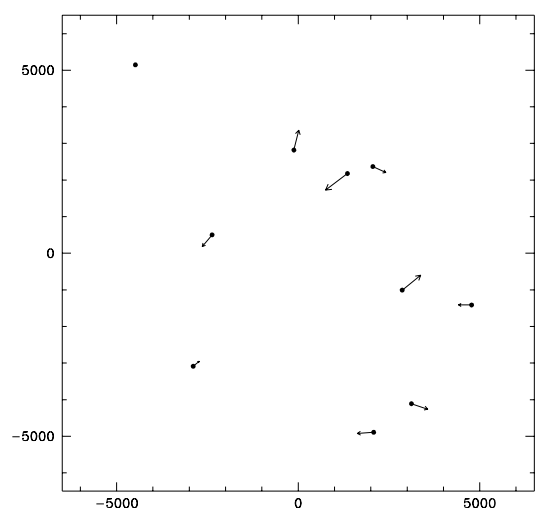

Fig. 10. Residuals for the mean position of the three exposures after the plate reduction with the PPM catalog as a function of the plate position. Arrow lengths have been enlarged 1500 times

\section{Photometry}

An internal instrumental magnitude was computed from the measured density of each exposure. For this purpose, 
the volume under each Gaussian was considered and an internal instrumental magnitude was defined as

$m_{k}=-2.5 \log \left(v_{k}\right)$,

where $v_{k}=A_{k}\left(\pi a_{k} b_{k}\right), A_{k}$ being the peak density of the $k$-th exposure in the triple image, and $a_{k}, b_{k}$ the semiaxes of the elliptical contour. But this is true only in the case of non-saturated images for which a Gaussian model is correct.

For saturated images it happens that the volume of the fitted Gaussian is smaller than the one which would correspond to a linear detector without saturation because the image is, in some way, cut by the saturation limit of the plate.

Let us suppose that we have a Gaussian and that we cut it in two parts at a certain height $h$. The ratio between the volume of the "lower" part, $V$, and the total one, $V_{\mathrm{c}}$, is found to be:

$$
\frac{V}{V_{\mathrm{c}}}=h \times(1-\ln h)
$$

where $h$ is the truncation height and the total height is assumed to be 1 .

This allows to obtain the "true" Gaussian volume $V_{\mathrm{c}}$ in the case of saturated (truncated) images as a function of the plate saturation peak (truncation) $p_{\mathrm{S}}$, the image area $a$, and the measured volume $V$ :

$V_{\mathrm{c}}=a \times p_{\mathrm{S}} \times \exp \left\{\frac{V}{a \times p_{\mathrm{S}}}-1\right\}$.

This $V_{\mathrm{c}}$ is an estimation of the volume which can be used in the evaluation of the internal magnitude when dealing with saturated images. The measured volume $V$ is a Gaussian one, corresponding to the volume under a bivariate Gaussian: It is the semimajor times the semiminor image axis times maximum amplitude of image peak density. We are interested also in comparing the magnitudes estimated from $V$ (uncorrected) and the ones from $V_{\mathrm{c}}$ (corrected).

The photometric reduction was performed with 102 stars from the SIMBAD data base which have been identified in the plate. More than $70 \%$ of these data comes from a work by Eggen \& Sandage (1964), which ensures that we are dealing with a mostly homogeneous sample.

A second order polynomial was used to fit the data and accuracies of $\sigma= \pm 0^{\mathrm{m}} .09$ (case of uncorrected magnitudes) and $\sigma_{\mathrm{c}}= \pm 0^{\mathrm{m}} .10$ (when corrected magnitudes are introduced) were obtained. A total of two outliers and seven poor quality stars were removed from the sample prior to the fitting. An analysis of these poor quality stars revealed that six of them are the brightest stars in the sample located at a distance from the plate center larger than $4.7 \mathrm{~cm}$, where optical aberrations produce important distortions on the images. The seventh one removed is the brightest star in the whole sample. As the method

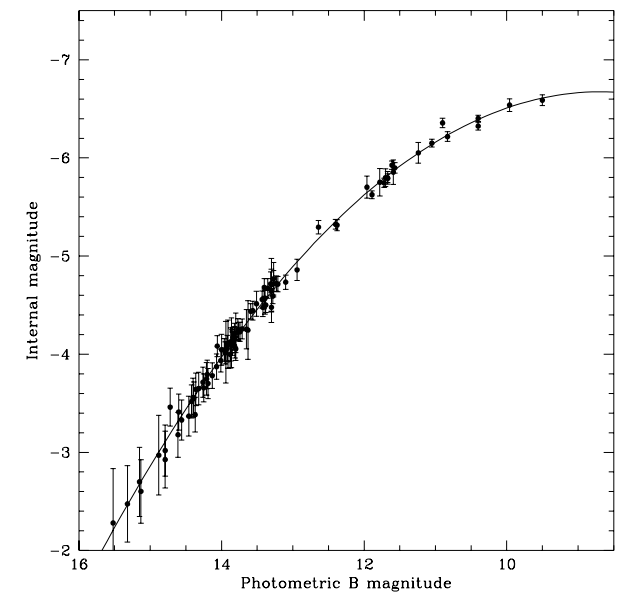

Fig. 11. Magnitude calibration using the measured internal magnitudes and a photometric sequence

of corrected volumes relies on the proper estimation of the image area, it is very sensitive to distorted cases such as the ones just described. Thus, a wrong estimation of the image area produces a wrong estimation of the correction to the image volume and this method cannot deal with these pathological cases. The method of corrected magnitudes constitutes an experimental model which forces the calibration curve to be closer to linear at the bright end and may be used if such a property were essential. In Fig. 11 we show how the fitting looks like when using uncorrected magnitudes, after dropping off the seven problematic cases. The error bars in this figure are the propagated errors of the profile parameter errors.

One can compare the accuracy obtained here, $\sigma=$ $\pm 0^{\mathrm{m}} .09$ with the accuracy in Eggen \& Sandage paper, which is $\sigma_{\mathrm{ES}} \approx \pm 0^{\mathrm{m}} .02$. It is clear that $\sigma_{\mathrm{ES}}^{2}$ is negligible in comparison with $\sigma^{2}$, therefore one can state that the main sources of the found rms error $\sigma$ must be related to the quality of our $\mathrm{CdC}$ plate and to the specific reduction method described here.

\section{Reducing the number of free parameters in the model}

A problem present in the three-gaussian model fitted to the data is the large number of free parameters, 22 in total per triple image. It is clear that some of these parameters must be correlated as the three exposures were intended to be as similar as possible. Therefore a model with smaller number of degrees of freedom would be interesting to consider because:

a) The fitting process would be more robust with a smaller number of free parameters.

b) The fitting algorithm would be faster.

Principal Components Analysis (PCA) (Brosche 1973; Whitney 1983, for example) is a method which first obtains a new set of uncorrelated variables by 
determining the set of eigenvectors of the original data correla-

tion matrix. The corresponding eigenvalues give the portion of total variation in the original data for which each eigenvector accounts for. Then the eigenvalues are sorted and split into two groups: The ones with larger values putatively represent the signal, and the remaining ones represent the noise. The programme used (Lentes 1985) contains a modification of the correlation matrix in order to take care for error covariance.

We found that it is possible to reduce the number of free parameters to not less than 7 . This minimum is defined by the number of eigenvalues with larger values representing the actual data.

A question can be posed now: Is there any set of less than 22 parameters which seems suitable a priori to represent the data? Some characteristics in our problem can help us to find a possible candidate. For example, the three exposures should in principle be identical, as they were supposed to be given the same exposure time in each individual plate and were taken under approximately the same atmospheric conditions, as there was only about half an hour between consecutive exposures. More explicitly, we have found that, in average, in the current data the values of the parameters in an individual exposure are compatible with the case of being equal to the analogue ones in the other two exposures for each single star. This means that it is basically correct to use only one peak density value $A$ for the three exposures, and the same holds for $\sigma_{x}, \sigma_{y}, s$ and $t$. The only parameter left, the background, was found to be varying in a not at all regular way over the plate (Fig. 12). However, the scale of this variation is much larger than just the $0.81 \times 0.81 \mathrm{~mm}$ windows containing the triple images and no errors are introduced when taking it to be constant. Thus, it was considered to be the same inside the whole frame containing the triple image

The reasonings above, thus, lead in total to a set of 12 free parameters, that is, the 6 mentioned just before and 6 position coordinates for the centres of the three exposures. These have been the degrees of freedom of the new model applied in Sect. 7.

The question remains whether it is possible to only have 7 degrees of freedom without loosing an important fraction of the original information. First, one would expect a strong correlation between $\sigma_{x}$ and $\sigma_{y}$ (and it is so indeed) hence one of them can be computed as a function of the other one. One has to pay attention to the fact that because of the presence of field curvature this function will depend on the distance to the plate centre.

Moreover, could it be possible to know the position of each exposure only knowing the triangle central coordinates? For this to be correct the triangle geometry should be constant through the plate. The angles which the position vectors of each exposure in a triplet form with an $x$-axis parallel to the $x$ plate scan axis and as origin the triangles geometric centre have been calculated, together with their rms. Its dependence on the position in the plate and on the distance to the plate centre was studied and it was found that exposure 1 suffers a displacement when approaching one of the plate corners. It also happens to exposure 3 , but to a smaller degree. The total dispersion of the angle values is equivalent to an image displacement along an arc of length of $00^{\prime \prime} 12,0$.' 03 and $00^{\prime \prime} 005$ for exposures 1, 2 and 3, respectively. One can compare this with the residuals when performing the comparison with an external catalogue (Table 3 ). If the total error in the image position is taken to be $\Delta d=\sqrt{(\sigma(\Delta x))^{2}+(\sigma(\Delta y))^{2}}$, we obtain $\Delta d=0^{\prime \prime} .30$, which is larger than $0^{\prime \prime} .12$. Thus, angle dispersion is not the main source of error in the position in this case. We have also checked that the ratios among the triangle sides are not dependent on the magnitude.

A model for the three exposure positions as a function only of the geometrical triangle centre position in the plate is then possible and this would lead to a set of 7 free parameters, as predicted by the PCA: $A, \sigma_{x}$ (or $\left.\sigma_{y}\right), s, t$, $x_{\mathrm{c}}, y_{\mathrm{c}}$ and the background $(B)$.

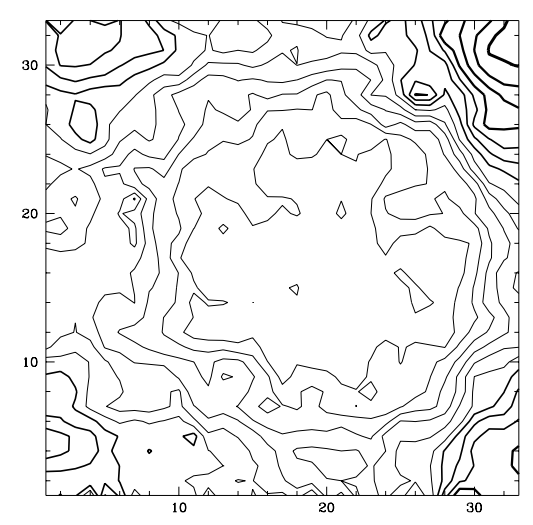

Fig. 12. Contour plot of different background levels. Thicker lines correspond to larger values. The non-uniformity of the background across the plate is obvious, as well as the large inhomogeneity toward the plate edges

\section{Reduction with a 12 free parameter model}

A new reduction was then performed, modeling the data now with a 12 parameter model instead of the 22 parameter one used before. This new model was simply the addition of three Gaussian curves with identical $A, \sigma_{x}, \sigma_{y}$, $s, t$ and $B$, together with the corresponding 6 coordinates for the three image centres:

$$
\begin{aligned}
D_{i j}= & B+\sum_{k=1}^{3} A \exp \left\{-\frac{1}{2}\left[\frac { 1 } { 1 - t ^ { 2 } } \left(\left(\frac{x_{i j}-x_{c k}}{\sigma_{x}}\right)^{2}\right.\right.\right. \\
& \left.\left.\left.+\left(\frac{y_{i j}-y_{c k}}{\sigma_{y}}\right)^{2}-2 t\left(\frac{x_{i j}-x_{c k}}{\sigma_{x}}\right)\left(\frac{y_{i j}-y_{c k}}{\sigma_{y}}\right)\right)\right]^{s}\right\}
\end{aligned}
$$




\subsection{Plate-to-plate transformation}

An internal comparison of the plate was again performed following the same method already described. There is an improvement in comparison with the results obtained when using the 22 free parameter model, as can be seen in the fourth row in Table 2. We noted that $\sigma(\Delta x)>\sigma(\Delta y)$, again due to border effects as we obtained $\sigma(\Delta x)=\sigma(\Delta y)=0$.' 10 when working with a circular area of radius $5 \mathrm{~cm}$ around the plate centre (note that it is the same obtained for the same region with the 22 parameter model). It seems from Table 2 that the 22 free parameter model was able to deal with this trend when considering the whole plate while the 12 parameter model was not. This means that distortions in the plate borders introduce significant deviations from the hypothetical identity of the three exposures, which is assumed in the 12 parameter model.

\subsection{Plate-to-catalogue reduction}

A remarkable improvement is obtained when working with the 12 parameter model when performing the reduction with the PPM as before (see last row in Table 3). Again, $\sigma(\Delta x)>\sigma(\Delta y)$ due to the poor quality of the reference stars and possible telescope tracking errors, as explained in Sect. 4.2 .

\subsection{Photometry}

A second order polynomial has been fitted to the data as before, after removing from the sample the seven ones which were found to be to severely affected by saturation and optical aberrations (see Sect. 5). The magnitudes are obtained with an accuracy of $\pm 0^{\mathrm{m}} \cdot 10$, to be compared with the one obtained with the 22 parameter model, $\pm 0^{\mathrm{m}} .09$. It is clear that both methods lead to the same accuracy in the photometry.

\section{Conclusions}

We showed that it is possible to make use of the information stored in the $\mathrm{CdC}$ plates in spite of the large variety of problems that they exhibit (triple exposures, optical aberrations, grid lines, emulsion saturation).

A general procedure in their analysis has been developed, based in the fitting to these triple exposures of a model consisting on the sum of three bi-variate Gaussian distributions in arbitrary position.

A detailed analysis of some problems in the plates was carried out. It was found that optical aberrations which result in symmetrically elongated images (spherical aberration and field curvature) dominate others which could be also present and would introduce asymmetries in the images (coma). We have seen that these particular aberrations have no influence in the final astrometric accuracy as the bivariate Gaussians are able to correctly model these elliptical images present mainly in the plate corners.

The internal plate accuracies obtained per exposure are $\sigma(\Delta x)= \pm 0$ "' 15 and $\sigma(\Delta y)= \pm 0$ "' 12 . Improvement is obtained when considering only a circular area around the plate centre as in this case accuracies were $\sigma(\Delta x)=\sigma(\Delta y)= \pm 0^{\prime \prime}$. 10 for both the 22 and 12 parameter models. This demonstrates the poorer quality of images at the plate borders due to optical distortions mainly.

The reduction with an external catalogue (PPM) gives values of $\sigma(\Delta x)= \pm 0$ ". 16 and $\sigma(\Delta y)= \pm 0$ "' 13 . This reduction was done with problematic data as all the stars considered were among the brightest ones on the plate and they exhibited larger errors in the fitting due to the blending of the three exposures. Only 10 stars could be used as reference stars. The differences among these $\sigma(\Delta x)$ and $\sigma(\Delta y)$ are most probably due to the poorer quality of the bright images and/or problems in the telescope tracking.

The 12 free parameter model is found to be equivalent to the 22 parameter model for the internal reduction but for the external reduction results are better with the first one. This is because although the 22 parameter model is capable of reproducing the data in a more detailed way it is also more sensitive to individual image errors.

A photometric reduction was also performed using a homogeneous sample of data from Eggen \& Sandage (1964) mainly, with a resulting accuracy of $0^{\mathrm{m}} .09$. Photometry with the 12 parameter model gives the same result, so the photometry is not affected by the change in the model in these two particular cases.

Improvements to the algorithm have been also outlined on the basis of the results obtained with a Principal Component Analysis of the data which indicates that a model with at least 7 free parameters instead of 22 is possible. These parameters are: one peak density $(A)$, one profile width $\sigma$, one saturation parameter $(s)$, one orientation parameter $(t)$, one background $(B)$ and the triangle centre coordinates $\left(x_{\mathrm{c}}, y_{\mathrm{c}}\right)$.

Acknowledgements. The authors wish to thank Drs. H.-J. Tucholke and M. Geffert for providing us with part of the software used in this work, together with Dr. M. Odenkirchen, Dr. A. Fernández-Soto and Prof. Dr. K.S. de Boer for very useful discussions and comments. Dr. Th. Lentes provided us with his software to perform the Principal Components Analysis. We are very grateful to Bordeaux Observatory for providing us several CdC plates from their archives. We also thank SIMBAD Data Base at the CDS (Strasbourg, France) for providing us part of the data used.

This work has been carried out in the frame of a European Community (EC) Network entitled "Salvaging an astrometric treasure" (project no. CHRX-CT94-0533) within the "Human and Capital Mobility" program. The authors duly acknowledge the support of the EC, specially A. Ortiz-Gil and M. Hiesgen with regards to their fellowships. 


\section{References}

Bonnefond P., 1991, DEA Thesis, Observatoire de Paris Brosche P., 1973, A\&A 23, 259

Dick W.R., Tucholke H.-J., Brosche P., Galas R., Geffert M., Guibert J., 1993, A\&A 279, 267

Eggen O.J., Sandage A.R., 1964, ApJ 140, 130

Geffert M., Bonnefond P., Maintz G., Guibert J., 1996, A\&AS 118,277

Girard T.M., Grundy W.M., López C.E., Van Altena W.F., 1989, AJ 98, 227

Hiesgen M., Brosche P., Ortiz-Gil A., 1996, AG Abstr. Ser. 12, 240

Hiesgen M., et al. (in preparation)

Horstmann H., 1988, in: Seitter W.C., Duerbeck H.W., Tacke M. (eds.), Large-Scale Structures in the Universe, Observational and Analytical Methods, Lecture Notes in Physics, 310. Springer, Berlin, Heidelberg, p. 111
Kostinsky S., 1907, Mitt. Nikolai-Hauptsternwarte, Pulkovo, Band II, 14

Lentes Th., 1985, Dissertation at the University of Bonn, Germany

Marquardt D.W., 1963, J. Soc. Ind. Appl. Math. 11, 431

Ortiz-Gil A., Brosche P., Hiesgen M., 1995, AG Abstr. Ser. 11, 92

Potter H., Polyakov E., Kopylov I., Kanayev I., Evdokimov A., López A., Flores A., Zalles R., 1996, New observational projects, in: López A., Yagudina E.I., Martínez Usó M.J., Cordero Barbero A. (eds.) Third International Workshop on Positional Astronomy and Celestial Mechanics. Observatorio Astronómico Universidad de Valencia, Valencia (Spain), p. 19

Ross F.E., 1921, ApJ 53, 5

Urban S.E., Corbin T.E., 1996, A\&A 305, 989

Whitney C.A., 1983, A\&AS 51, 443 